\title{
ANALYSIS OF MICROTUBULE NUMBER AND LENGTH IN CYTOSKELETONS OF CULTURED CHICK SENSORY NEURONS ${ }^{1}$
}

\author{
PAUL C. LETOURNEAU ${ }^{2}$
}

Department of Anatomy, University of Minnesota, Minneapolis, Minnesota 55455

Received September 22, 1981; Revised February 8, 1982; Accepted February 10, 1982

\begin{abstract}
In order to examine the continuity of individual microtubules in growing neurites, microtubules were observed and counted in whole mounted cytoskeletons of chick embryo sensory neurons. At neurite branch points, the microtubules of the proximal segment are divided between the distal segments without the addition of microtubules to the distal branches. Few microtubule ends were seen in the cytoskeletons, and on occasion, the same number of microtubules was counted at widely separated points along a neurite segment.

I conclude that microtubules are long in embryonal neurites, and some may extend the entire length from perikaryon to neurite tip. This may limit where microtubule growth occurs to the perikaryon and the neurite tip, where many microtubule ends are. In addition, the great length of most neuritic microtubules may explain why collateral sprouting is rare along embryonal neurites.
\end{abstract}

The microtubules in nerve axons (Palay and ChanPalay, 1977) may be important in supporting axonal structure and in transporting materials (James et al., 1970; Lasek and Hoffman, 1976; Ochs and Worth, 1978; Weiss and Mayr, 1971). Because uninterrupted microtubules would be effective tracks for axonal transport, several studies have asked whether individual microtubules span the entire length of adult axons. Earlier work (Nadelhaft, 1974; Weiss and Mayr, 1971) found that the sum of the microtubules in distal neurite branches equals the number in a proximal parent segment (but see Zenker and Hohberg, 1973). In addition, the kinetics of tubulin movement suggest that little microtubule assembly and disassembly occurs in axons (Black and Lasek, 1980; Lasek and Hoffman, 1976). However, these findings are not direct determinations of microtubule length in axons. In more recent analyses, axonal segments up to 10 to 15 $\mu \mathrm{m}$ were serially sectioned, and the frequency of microtubule ends indicated that the average microtubule exceeds $100 \mu \mathrm{m}$ (Bray and Bunge, 1981; Tsukita and Ishikawa, 1980). Yet, the presence of microtubule ends indi-

\footnotetext{
' I thank Alice Ressler for expert technical work in the analyses of microtubule number and the preparation of the electron micrographs. The secretarial skills of Roberta Andrich were appreciated also. This research was supported by the National Science Foundation and by an American Cancer Society Institutional Grant to the University of Minnesota.

${ }^{2}$ To whom correspondence should be addressed at Department of Anatomy, 4-135 Jackson Hall, University of Minnesota, 321 Church Street, S.E., Minneapolis, MN 55455.
}

cated that many microtubules are wholly contained within an axon (Bray and Bunge, 1981; Chalfie and Thomson, 1979; Tsukita and Ishikawa, 1980).

This paper is concerned with the role that microtubules may have in the development of the shapes of neurites of embryonal neurons. Neurite branches usually arise by the division of a growing nerve tip (Bray, 1973), and collateral sprouting proximal to the tip is rare, even though proximal neurite segments can be induced to form growth cones (Bray et al., 1978). Bray's findings may be related to the continuity and growth of microtubules in growing neurites. The formation of neurite-like processes by neuroblastoma cells is preceded by the aggregation of several microtubule initiation sites into a single initiation center (Osborn and Weber, 1977; Spiegelman et al., 1979). Microtubules originating from this center may continue to elongate at their distal ends (Heidemann et al., 1981) as a process grows. Thus, individual microtubules may extend from the perikaryon to the tips of growing neurite-like processes. If this were true in embryonal neurites, then collateral sprouting may be quite infrequent, because the initiation of new microtubules to supply a collateral sprout is infrequent along neurites.

Therefore, I wanted to examine microtubule continuity in embryonal neurites, paying special attention to branch points. I analyzed microtubules in the cytoskeletons of whole mounted embryonal neurons in order to examine large portions of the neuritic trees of embryonal neurons. Although some details were obscured in the cytoskeletons, the data suggest that microtubules 
are long in embryonal neurites, and at branch points, microtubules of the parent segment pass into the daughter branches without breaking or adding new microtubules.

\section{Materials and Methods}

Nerve cell culture. Sensory ganglia were removed from 8- to 11-day chick embryos and dissociated with trypsin as previously described (Letourneau, 1975). The resultant cells were placed into a medium of $50 \%$ Ham's F-12 (Gibco) containing 10\% fetal calf serum (Gibco) and 50\% heart-conditioned medium as prepared by Helfand et al. (1976). Nerve growth factor (a gift from Dr. Eric Shooter, Department of Neurobiology, Stanford University School of Medicine, Stanford, CA) was added at $10 \mathrm{ng}$ / $\mathrm{ml}$. Six $\times 10^{4}$ cells in $1.5 \mathrm{ml}$ of medium were plated in plastic Petri dishes (Falcon 1008) which contained gold electron microscopic grids (50 mesh; Ted Pella, Inc.) supporting a polyornithine-treated, carbon-coated $0.5 \%$ Formvar film (Letourneau, 1979). The dishes were incubated at $37^{\circ} \mathrm{C}$ in a humidified, $5 \% \mathrm{CO}_{2}$ incubator.

Preparation of cytoskeletons. A microtubule-stabilizing buffer (MtSB) was made containing $4 \%$ polyethylene glycol, $1 \mathrm{~mm}$ EGTA, $1.5 \mathrm{~mm}$ guanosine triphosphate, and 0.1 м HEPES buffer, $\mathrm{pH} 6.9$ (Webster et al., 1978). After $24 \mathrm{hr}$, the cell cultures were removed from the $\mathrm{CO}_{2}$ incubator, rinsed with serum-free Ham's F-12 medium and with MtSB. The neurons then were extracted by incubation in MtSB containing $0.4 \%$ Triton X-100 for 5 min at room temperature. The cultures were fixed by the addition of an equal volume of MtSB containing $4 \%$ glutaraldehyde. Fixation continued for $30 \mathrm{~min}$ at room temperature, and then the dishes were rinsed three times with Dulbecco's phosphate-buffered saline, followed by distilled water.

Electron microscopy. The cytoskcletons were stained with $2 \%$ aqueous uranyl acetate for 30 min, rinsed in $\mathrm{H}_{2} \mathrm{O}$, and dehydrated through $15,25,50,75,90$, and $100 \%$ acetone (Letourneau, 1979). The grids were dried in a critical point apparatus (Sorvall), and the cytoskeletons were lightly carbon coated. Specimens for scanning electron microscopy were sputter coated with gold/palladium. Transmission electron microscopy was done with a Philips 201C electron microscope at 60 or $80 \mathrm{kV}$, and scanning electron microscopy was done with a JEOL $100 \mathrm{C}$ microscope equipped with an ASID-4C high resolution apparatus.

Microtubule analysis. Microtubules were counted on prints of negatives photographed at $\times 10,500$ magnification and enlarged to $\times 40,000$ to 80,000 . The narrower neurofilaments were easily distinguished from microtubules (Fig. 5). A line was drawn roughly normal to the axis of a neurite and the microtubules intersecting the line were counted. At neurite branch points, counts were made within $20 \mu \mathrm{m}$ of the site of microtubule divergence.

\section{Results}

\section{Cytoskeleton of chick embryo sensory neurons}

Short term extraction of living cells with detergents has provided new structural information not apparent in thin sections of whole cells. Cytoskeletal organization in particular has been revealed in new ways, such as the interesting associations of cytoskeletal fibers with polysomes and plasmalemmal components (Ben-Ze'ev et al., 1979; Lenk and Penman, 1979), and detailed chemical and immunological analyses of the composition of cytoplasmic filaments have been performed (Brown et al., 1976; Webster et al., 1978).

The cytoskeleton of chick sensory neurons contains filaments and the nucleus (Figs. 1 and 2). The perikaryon is a tangle of microtubules and neurofilaments which have collapsed around the remains of the nucleus (Fig. 2). Clusters of particles associated with the filaments may be polysomes (Lenk and Penman, 1979), other organelles, or debris. At points of neurite origin, microtubules and neurofilaments are collected from the tangled filaments around the nucleus and pass down the neurites (Figs. 1 and 2). There is no evidence of distinct centers from which microtubules pass into each neurite, although these may have been obscured during extraction.

The microfilament network in the cell cortex and cytoplasmic matrix seen in thin sections of neurons (Bunge, 1973; Luduena-Anderson and Wessells, 1973; Yamada et al., 1971) is absent from these cytoskeletons, even at the neurite tip, which contains many microfilaments in unextracted cells (Letourneau, 1979; Yamada et al., 1971). Large microfilament bundles remain, however, in the non-neuronal cells of our extracted cultures (data not shown; see Spooner et al., 1971). The microfilaments of stress fibers contain proteins that may stabilize actin assembly (Lazarides, 1975, 1976). These proteins may not be associated with these neuronal microfilaments; therefore, they depolymerize rapidly when soluble actin is extracted. On the other hand, microtubules and neurofilaments seem resistant to the absence of soluble monomers for at least 20 min of extraction (Morris and Lasek, 1979).

\section{The neurite cytoskeleton}

In whole mounts of unextracted neurites, microtubules are seen as well as mitochondria and unidentified vesicles (Fig. 3). These organelles are embedded in a filamentous network that resembles the microtrabecular lattice of adult axons (Ellisman and Porter, 1980) and may correspond to the filamentous sidearms seen in thin sections of cultured chick sensory neurites (Yamada et al., 1971). However, neurite cytoskeletons contain only microtubules and neurofilaments (Figs. 4 and 5). The microtubules are long and relatively straight, but neurofilaments are disrupted from the straight course that they have in situ.

At neurite branch points, the cytoskeletal fibers of the parent neurite are diverted into the daughter branches (Fig. 4), and though microtubules sometimes trace a circuitous route from the parent into the daughter branches (Figs. 6 and 7), only once among 31 branch points examined did a microtubule seem to pass from one daughter branch to another daughter branch. Also evident at branch points was the winding of microtubules around each other (Figs. 8 to 10). This configuration probably results from collapse of the cytoskeleton during extraction but may reflect a helical twist of the microtubules as they pass down the neurite (Bray and Bunge, 

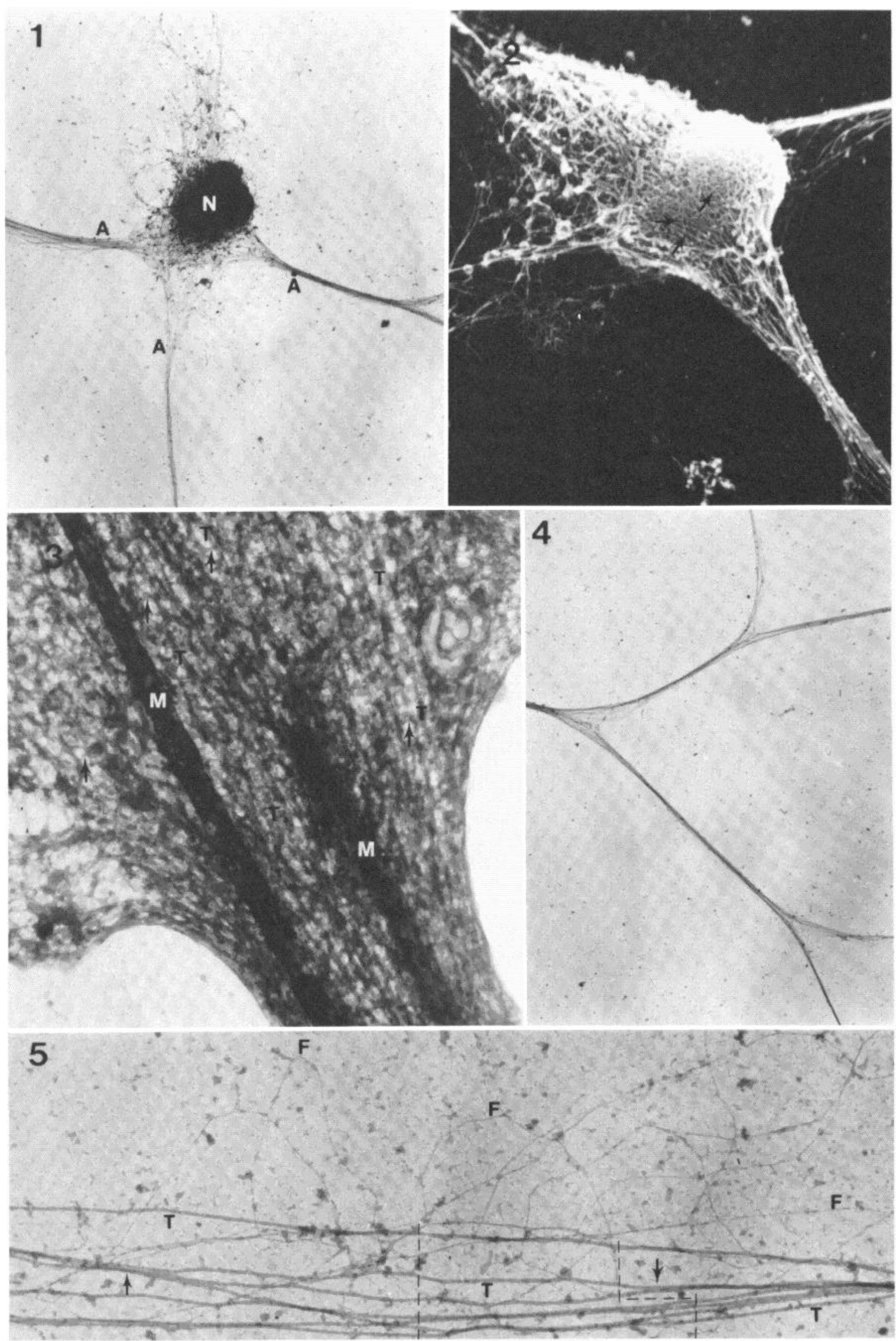

Figures 1 to 5

Figure 1. The perikaryon and proximal neuritic segments of the cytoskeleton of a detergent-extracted sensory neuron. Microtubules converge within the perikaryon and pass down the individual neurites $(A)$. $N$, Nucleus. Magnification $\times 1,500$. 
1981; Gilbert, 1975; Heacock and Agranoff, 1977; Metuzals and Izzard, 1969). A helical organization of cytoskeletal fibers may strengthen the cytoplasmic matrix of the neurite.

Numerical analysis. The major difficulty in counting microtubules in whole mounts was that microtubules were often closely packed (for example, Figs. 1, 4, and 8). Though many neuronal cytoskeletons were examined in the electron microscope, most were rejected, because the microtubules were aggregated or neurites were overlapping. From 15 grids containing hundreds of neuronal cytoskeletons, 9 neurons were chosen and photographed for numerical analysis. In these cytoskeletons, arrays of microtubules often were separated into individuals that could be counted without great difficulty. Perhaps these more separated arrays of microtubules had occupied adherent portions of neurites that were spread onto the polylysine-treated substratum (Letourneau, 1975, 1979). In good fortune, the microtubules often were well separated at neurite branch points. I expect that the counts underestimate microtubule number in areas of close packing, such as near the perikaryon, as in Figures 14 and 15 . These numbers, though inaccurate, do illustrate trends in microtubule number in proximal regions compared to the distal portions of the neuritic trees. In distal branches where microtubules are fewer and more easily seen, the numbers are more likely to be accurate. Figures $5,11,12$, and 22 illustrate regions where microtubules were counted for my analysis. Figures 11 and 12 were photographed at points $140 \mu \mathrm{m}$ apart along the same neurite cytoskeleton. Schematic drawings of the cytoskeletons and the microtubule counts of selected points are displayed in Figures 13 to 21 . I found that the conservation law of microtubule numbers (Weiss and Mayr, 1971) is upheld at neurite branch points, and more significantly, the law holds very well for the entirety of simple neuritic trees (Figs. 16 to 19).

Microtubules at neurite branch points. At a neurite branch point, the microtubules of the proximal neurite diverge and enter the distal or daughter branches without breaking so that the sum of the microtubules in the daughters tends to equal the number in the parent neurite (Fig. 22). Even if one daughter branch received many more microtubules than another, no daughter branch ever had an equal or greater number of microtubules than its parent segment at the point of branching. That is to say, branch points are not a place where many microtubules are added to the neurite. Beyond a branch point, microtubule number may not change or may rise or fall before the next branch point. Commonly, microtubule number decreased along the terminal unbranched segments of neurites. Despite this generalization, a few instances were noted where microtubule number at a neurite tip was much higher than proximally along the same segment (Figs. 15, $a$ and $b$, and 21, $a, b$, and $c$ ). Because the microtubules were well separated in these cases, these particular counts are accurate or very close to accurate.

Microtubule length. These photographs (Figs. 1 to 12 and 22) portray my observation that few microtubule ends are present along the cytoskeletons of neurites. This is expected if many microtubules are long, though some ends may be hidden in aggregates of microtubules. As expected, I did see many microtubule ends in the cytoskeletons of growth cones (Fig. 23). Both proximal and distal (relative to the perikaryon) microtubule ends were seen along neurite cytoskeletons, suggesting, as our counts did (Figs. 15, $a$ and $b$, and $21 a, b$, and $c$ ), that some microtubules are present only in distal neurite segments. Estimates of microtubule length could be made from the frequency of microtubule ends visible in the cytoskeletons. For example, Figure 22 contains a minimum of $175 \mu \mathrm{m}$ of uninterrupted microtubules with no visible breaks. Since I am not sure that I see all microtubule ends, such estimates were not calculated.

Some ends might have occurred by breakage of some microtubules during extraction, fixation, or later. Though some ends were thinned, indicating partial depolymerization of microtubules, even after $20 \mathrm{~min}$ of extraction, few microtubule ends were seen along neurite cytoskeletons. It should be realized that microtubule breaking during fixation or later also may have occurred in the preparation of axons for conventional thin sectioning (Bray and Bunge, 1981; Chalfie and Thomson, 1979; Tsukita and Ishikawa, 1980).

Further indirect evidence of long microtubules was provided by several observations of the same number at widely separated points along individual neuritic segments (Figs. 11, 12, 17, $a$ and $b$, and $18 a, b, c$, and $d$ ). Of course, these may just be, coincidentally, the same number at two points.

\section{Discussion}

These studies were undertaken because microtubule continuity has not been examined previously in actively growing neurites of embryonal neurons. Findings with nongrowing axons or the neurite-like processes of neuroblastoma cells may not fully apply to embryonal neurites undergoing morphological changes. In addition, axonal branch points were not examined by serial sectioning in previous work to establish whether microtubule ends

Figure 2. Scanning electron micrograph of the cytoskeleton of a neuronal perikaryon. Microtubules (arrows) are collapsed in a tangle around the cell nucleus. Magnification $\times 4,000$.

Figure 3. Neuritic segment from a whole mount of an unextracted sensory neuron. Microtubules $(T)$ and mitochondria $(M)$ are oriented along the axis of the neurite. These organelles are embedded in a matrix of short filamentous segments (arrows) that are absent in cytoskeletons. Magnification $\times 48,000$.

Figure 4. Cytoskeleton of a portion of a neuritic tree. Microtubules diverge at branch points to pass from the parent into the daughter segments. The perikaryon is to the left. Magnification $\times 1,500$.

Figure 5. Illustration of the distinction between neurofilaments $(F)$ and microtubules $(T)$ in cytoskeletons of neurites. The bundling and unbundling of microtubules (arrows), which confuses counting, is seen also, although 7 microtubules can be distinguished at several points (dashed lines). Magnification $\times 32,000$. 


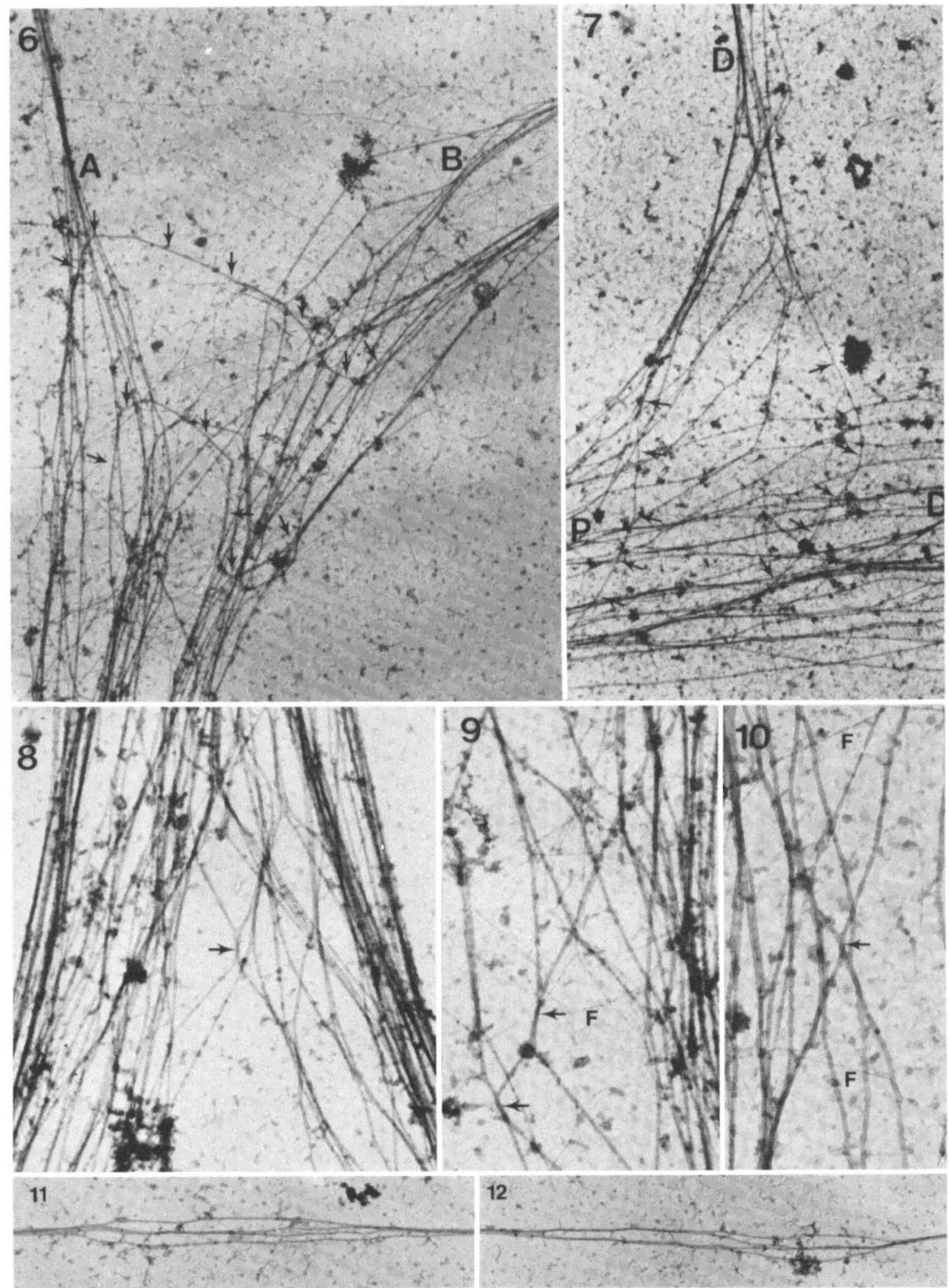

Figure 6. Divergence of microtubules at a neuritic branch point. Several microtubules (arrows) enter one distal branch (A) and then curve back and pass down the other daughter branch $(B)$. Magnification $\times 21,000$.

Figure 7. Divergence of microtubules at a neuritic branch point. Microtubules (arrows) sometimes cross over others while entering a distal branch. $P$, Proximal segment; $D$, distal segment. Magnification $\times 19,500$.

Figures 8, 9, and 10. Illustrations of microtubules twisted around one another in neuritic cytoskeletons. This is suggested by the apparent tensions exerted on the interacting microtubules at the points where they touch (arrows). $F$, Neurofilament. Magnification: Figure 8, $\times 30,000$; Figure 9, $\times 34,000$; Figure 10, $\times 43,000$.

Figures 11 and 12. Examples of the bundling and unbundling of microtubules along a neurite. These photographs were taken at two sites along the segment between points $c$ and $d$ in Figure 18. Magnification: Figure 11, $\times 22,000 ;$ Figure 12, $\times 17,000$. 

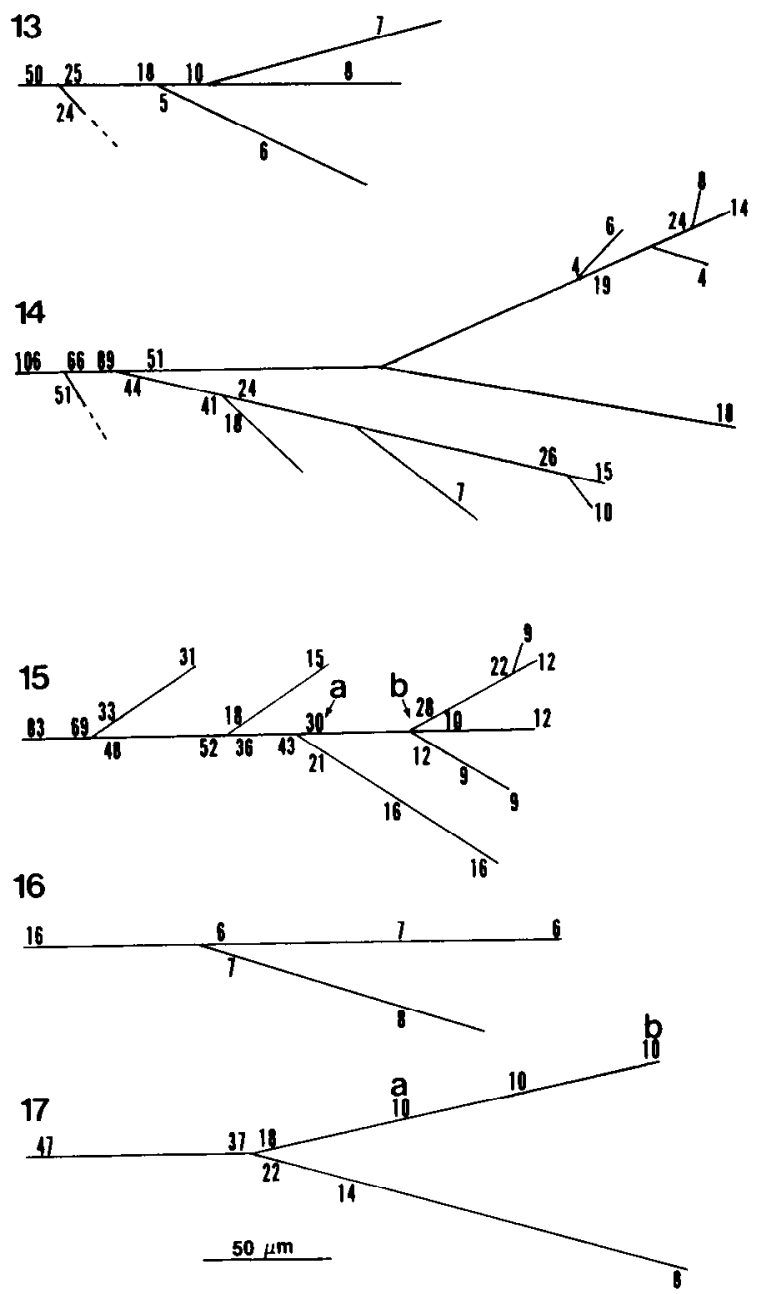
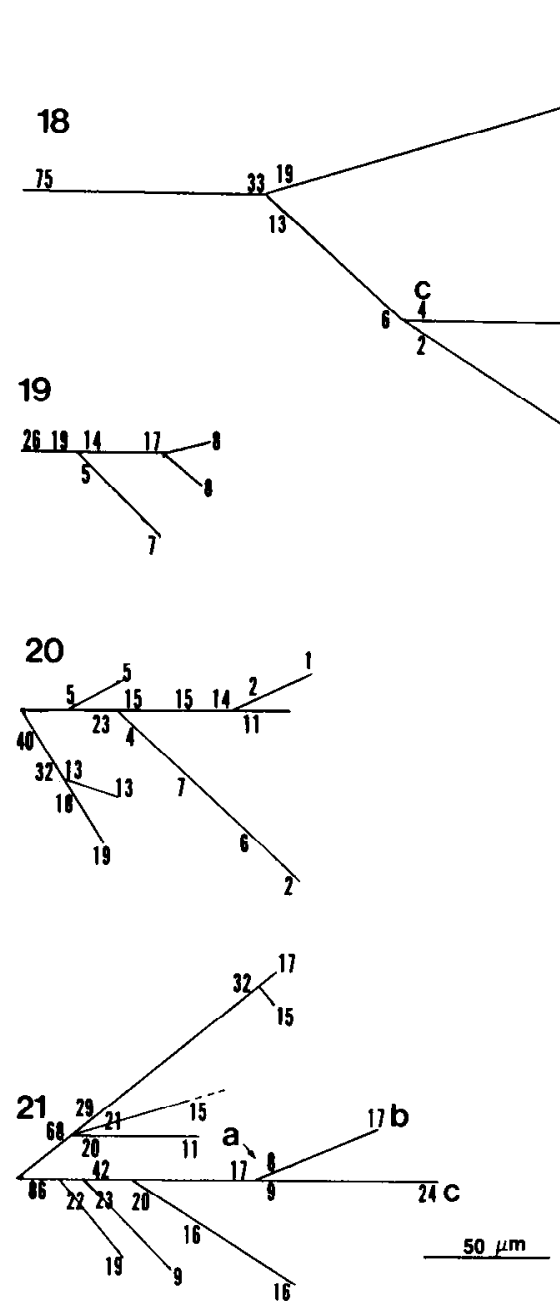

Figures 13 to 21 . Schematic drawings showing the lengths of segments in the neuritic cytoskeletons and the numbers of microtubules counted at selected sites. All drawings are at the same linear scale, although the actual course of the neuritic segments is not depicted. The perikaryon is located at the left side of each figure.

were present. The application of serial cross-sectioning to thin, single embryonic neurites would be exceedingly tedious, and especially challenging, if one wanted to examine several neurite branch points and reconstruct the courses of several microtubules. Hence, I used cytoskeletons of whole mounted embryonal neurons. Using extreme care to overcome the difficulty in completely following microtubules and their ends, 9 neuronal cytoskeletons were analyzed and microtubules were tracked through 31 branch points.

These findings suggest that microtubules are long in growing neurites. A mean length, calculated from the frequency of microtubule ends visible in our cytoskeletons, would be quite long. Yet, this may be an inaccurate estimate, if microtubule ends are hidden in aggregates along the neurite cytoskeletons. The numerical analysis, as well, is consistent with the presence of long microtubules, although the evidence is indirect. Thus, it seems that growing neurites resemble adult axons in the sense that some microtubules originate in the perikaryon and terminate short of neurite tips and other microtubules are present only distally in neurite segments. However, growing neurites may differ from adult axons in that many microtubules may extend continuously from the perikaryon to the tips of the shorter, narrow neurites of embryonal neurons. Also differing from at least one study of adult axons (Zenker and Hohberg, 1973), the sum of microtubules in a group of related distal branches is not several times the number present in the parent stem fiber (Figs. 13 to 21).

Before discussing the importance of microtubules to neurite branching, consider how microtubules get into growing neurites. If the faster growing ends of microtubules (Kirschner, 1980) are oriented distally, as in adult sympathetic axons (Heidemann et al., 1981), then tubulin could add onto the distal ends of microtubules as a neurite extends. In addition, new microtubules may be initiated in neurites. Of course, initiation or simple growth of microtubules in neurites requires that sufficient tubulin and initiation factors (Rosenbaum and McIntosh, 1976) be present to drive polymerization in growing neurites. Alternatively, completely assembled microtubules of any length may be transported without further growth from the perikaryon into neurites. Just as fishing line is transported from the end of a fishing rod by external force, long microtubules may be moved from the perikaryon down the length of neurites by mechanical forces, such as actomyosin interactions (Letourneau, 1981). Neither of these alternatives has been currently eliminated. 

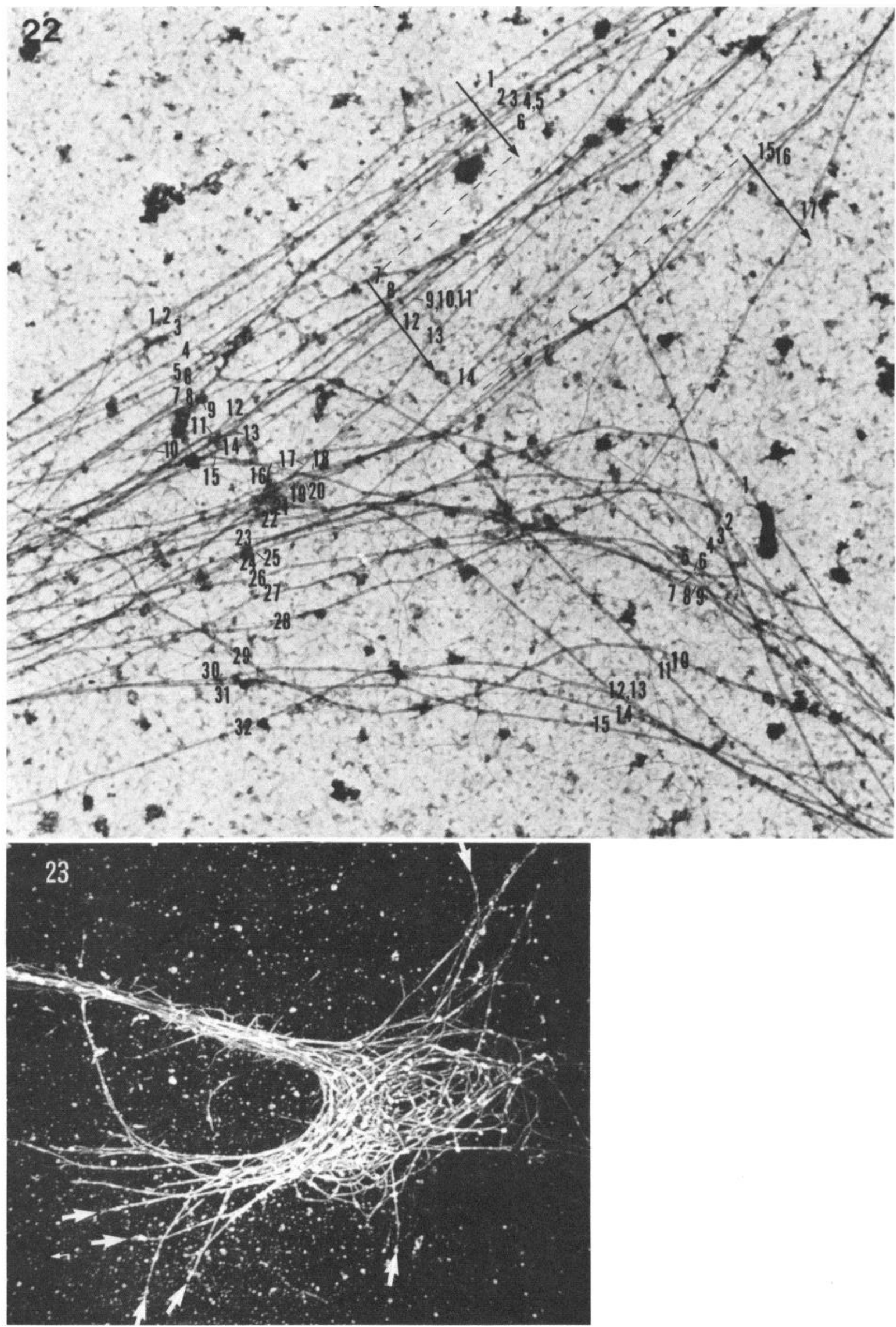

Figures 22 and 23 
These findings at branch points that microtubules are continuous and that additional microtubules are not added can explain Bray's observation (1973) that most neurite branches arise by bifurcation of the growing neurite tip rather than by the formation of collateral sprouts from the neurite trunk. Neurite segments generated by branching must be furnished with microtubules. This is easy in a bifurcating neurite tip, where microtubule ends can be diverted into the nascent branches (Fig. 23), possibly by the action of actomyosin-like forces (Letourneau, 1981). However, because microtubules in these growing neurites are long, there are few microtubule ends along a neurite to be diverted into a collateral sprout. The formation of collateral sprouts is limited further, because only the distal ends (relative to the perikaryon) of microtubules enter neurite branches. A sprout could be initiated, if new microtubules were assembled at one point in a neurite, but our counts and observations indicate that this does not commonly occur at branch points. Microtubule ends for a sprout could be generated by introducing breaks into existing microtubules, but this must be done such that the conservation law of microtubule number (Weiss and Mayr, 1971) continues to be upheld.

In summary, I propose that collateral sprouting is rare early in neurite outgrowth, because few microtubule ends are present along neurites except at the neurite tips, and microtubule ends are not commonly generated by other means. This feature may dictate that, in the early stages of neurite growth, branching morphogenesis is determined by the behavior of the neurite tip. This constraint on branching may be important in regulating the characteristic patterns of axonal and dendritic trees (Goodman, 1974, 1978). The generation of microtubules and the placement of microtubule ends also may be factors during regeneration of severed axons and, in the case of the sprouting of motor axons, in response to denervation of muscle fibers (Edds, 1953).

\section{References}

Ben-Ze'ev, A., A. Duerr, F. Solomon, and S. Penman (1979) The outer boundary of the cytoskeleton: A lamina derived from plasma membrane proteins. Cell 17: 859-865.

Black, M. M., and R. J. Lasek (1980) Slow components of axonal transport: Two cytoskeletal networks. J. Cell Biol. 86: 616-623.

Bray, D. (1973) Branching patterns of individual sympathetic neurons in culture. J. Cell Biol. 56: 702-712.

Bray, D., and M. B. Bunge (1981) Serial analysis of microtubules in cultured rat sensory axons. J. Neurocytol. 10: 589605.

Bray, D., C. Thomas, and G. Shaw (1978) Growth cone formation in cultures of sensory neurons. Proc. Natl. Acad. Sci. U. S. A. 75: 5226-5229.

Brown, S., W. Levinson, and J. A. Spudich (1976) Cytoskeletal elements of chick embryo fibroblasts revealed by detergent extraction. J. Supramol. Struct. 5: 119-130.

Bunge, M. B. (1973) Fine structure of nerve fibers and growth cones of isolated sympathetic neurons in culture. J. Cell Biol. 56: 713-725.

Chalfie, M., and J. N. Thomson (1979) Organization of neuronal microtubules in the nematode, Caenorhabditis elegans. J. Cell Biol. 82: 278-289.

Edds, M. V. (1953) Collateral nerve regeneration. Q. Rev. Biol. 28: $260-276$.

Ellisman, M. H., and K. R. Porter (1980) Microtrabecular structure of the axoplasmic matrix: Visualization of crosslinking structures and their distribution. J. Cell Biol. 87: 464479.

Gilbert, D. S. (1975) Axoplasmic architecture and physical properties as seen in the Myxicola giant axon. J. Physiol. (Lond.) 253: 257-319.

Goodman, C. S. (1974) Anatomy of the locust ocellar interneurons: Constancy and variability. J. Comp. Physiol. 95: 185201.

Goodman, C. S. (1978) Isogenic grasshoppers: Genetic variability in the morphology of identified neurons. J. Comp. Neurol. 182: 681-706.

Heacock, A. M., and B. W. Agranoff (1977) Clockwise growth of neurites from retinal explants. Science 198: 64-68.

Heidemann, S. R., J. M. Landers, and M. A. Hamborg (1981) Polarity orientation of axonal microtubules. J. Cell Biol. 91: 661-665.

Helfand, S. L., G. A. Smith, and N. K. Wessells (1976) Survival and development in culture of dissociated parasympathetic neurons from ciliary ganglia. Dev. Biol. 50: 541-547.

James, K. C., J. J. Bray, I. G. Morgan, and L. Austin (1970) The effect of cholchicine on the transport of axonal protein in the chicken. Biochem. J. 117: 767-771.

Kirschner, M. W. (1980) Implications of treadmilling for the stability and polarity of actin and tubulin polymers in vivo. J. Cell Biol. 86: 330-334.

Lasek, R. J., and P. N. Hoffman (1976) The neuronal cytoskeleton, axonal transport and axonal growth. In Cell Motility, R. Goldman, T. Pollard, and J. Rosenbaum, eds., pp. 1021-1049, Cold Spring Harbor Laboratory, Cold Spring Harbor, NY.

Lazarides, E. (1975) Tropomyosin antibody: The specific localization of tropomyosin in nonmuscle cells. J. Cell Biol. 65: 549-561.

Lazarides, E. (1976) Two general classes of cytoplasmic actin filaments in tissue culture cells: The role of tropomyosin. $J$. Supramol. Struct. 5: 531-563.

Lenk, R., and S. Penman (1979) The cytoskeletal framework and poliovirus metabolism. Cell 16: 289-301.

Letourneau, P. C. (1975) Possible roles for cell-to-substratum adhesion in neuronal morphogenesis. Dev. Biol. 44: 77-91.

Letourneau, P. C. (1979) Cell-substratum adhesion of neurite growth cones, and its role in neurite elongation. Exp. Cell Res. 124: 127-138.

Letourneau, P. C. (1981) Immunocytochemical evidence for colocalization in neurite growth cones of actin and myosin and their relationship to cell-substratum adhesions. Dev. Biol. 85: 113-122.

Figure 22. A neurite branch point located at the upper right of the cytoskeleton depicted in Figure 21. the numbers indicate the position of the 32 microtubules of the parent segment and the 17 and 15 microtubules, respectively, of the daughter segments. This photograph illustrates the way that we counted microtubules, although it is slightly smaller than the enlargement used for the original counts. Magnification $\times 33,000$.

Figure 23. Cytoskeleton of a growth cone. Many ends of microtubules (arrows) are seen where they projected into the base of the growth cone. The microfilamentous network has been extracted. Magnification $\times 4,000$. 
Luduena-Anderson, M., and N. K. Wessells (1973) Cell locomotion nerve elongation and microfilaments. Dev. Biol. 30: 427-440.

Metuzals, J., and C. S. Izzard (1969) Spatial patterns of threadlike elements in the axoplasm of the giant nerve fiber of the squid (Loligo pealiil) as disclosed by differential interference microscopy and by electron microscopy. J. Cell Biol. 43: 456479 .

Morris, J. R., and R. J. Lasek (1979) Differential solubility of cytoskeletal proteins in squid axoplasm. Soc. Neurosci. Abstr. 5: 504 .

Nadelhaft, I. (1974) Microtubule densities and total number in selected axons of the crayfish abdominal nerve cord. J. Neurocytol. 3: 73-86.

Ochs, S., and R. M. Worth (1978) Axoplasmic transport in normal and pathological systems. In Physiology and Pathobiology of Axons, S. G. Waxman, ed., pp. 251-264, Raven Press, New York.

Osborn, M., and K. Weber (1977) The display of microtubules in transformed cells. Cell 12: 561-571.

Palay, S. L., and V. Chan-Palay (1977) General morphology of neurons and neuroglia. In Handbook of Physiology. Vol. I: The Nervous System, E. R. Kandel, ed., pp. 5-37, American Physiological Society, Bethesda, MD.

Rosenbaum, J., and J. R. McIntosh (1976) Section 10 and
Section 11. In Cell Motility, R. Goldman, 'T. Pollard, and J. Rosenbaum, eds., pp. 1065-1168, Cold Spring Harbor Laboratory, Cold Spring Harbor, NY.

Spiegelman, B. M., M. A. Lopata, and M. W. Kirschner (1979) Aggregation of microtubule initiation sites preceding neurite outgrowth in mouse neuroblastoma cells. Cell 16: 253-263.

Spooner, B. S., K. M. Yamada, and N. K. Wessells (1971) Microfilaments and cell locomotion. J. Cell Biol. 49: 593-613.

Tsukita, S., and H. Ishikawa (1980) Morphological bases of fast axonal transport. Eur. J. Cell Biol. 22: 348.

Webster, R. E., D. Henderson, M. Osborn, and K. Weber (1978) Three-dimensional electron microscopical visualization of the cytoskeleton of animal cells: Immunoferritin identification of actin- and tubulin-containing structures. Proc. Natl. Acad. Sci. U. S. A. 75: 5511-5515.

Weiss, P., and R. Mayr (1971) Neuronal organelles in neuroplasmic (axonal) flow. II. Neurotubules. Acta Neuropathol. (Berl.) Suppl. 5: 198-206.

Yamuda, K. M., B. S. Spooner, and N. K. Wessells (1971) Ultrastructure and function of growth cones and axons of cultured nerve cells. J. Cell Biol. 49: 614-635.

Zenker, W., and E. Hohberg (1973) An $\alpha$-nerve fibre: Number of neurotubules in the stem fibre and in terminal branches. J. Neurocytol. 2: 143-148. 